\title{
ON SIMULTANEOUS INTERPOLATION AND APPROXIMA- TION BY FUNCTIONS ANALYTIC IN A GIVEN REGION
}

\author{
BY \\ J. L. WALSH AND H. G. RUSSELL
}

1. Introduction. In 1938 Walsh [8]( $\left.{ }^{1}\right)$ commenced the study of interpolation and the study of approximation by functions assumed merely analytic and bounded in a given region, topics which were further considered later $[4,9,10,11,12]$ in collaboration with Nilson. It is the object of the present paper to study simultaneous interpolation and approximation by functions analytic and bounded in a given region, where the function approximated is not necessarily analytic and where there is no necessary connection between the latter function and the values interpolated to. Throughout this paper we use the following notation. In the z-plane let $R$ be a finite region whose boundary $C_{1}$ consists of a finite number of mutually disjoint Jordan curves. Let $S$ be a point set $\left.{ }^{2}\right)$ whose boundary $C_{0}$ consists of a finite number of mutually disjoint Jordan curves $C_{0}^{(j)}$, where $\bar{S}=S+C_{0}$ lies in $R$ and separates no point of $R-\bar{S}$ from $C_{1}$.

We proceed to study the following problems. Let there be given a finite number of fixed conditions of interpolation at points of $S$. Let $M(>0)$ be given, let $f_{M}(z)$ be analytic and of modulus not greater than $M$ in $R$, and let $f_{M}(z)$ satisfy the given conditions of interpolation. We study the convergence $(M \rightarrow \infty)$ of the set $f_{M}(z)$, where in Problem 1 the function $f_{M}(z)$ approximates best on $C_{0}$ to a given function $F(z)$ defined on $C_{0}$, and approximation is measured on each component of $C_{0}$ in the sense of Tchebycheff; in Problem 2 the function $f_{M}(z)$ approximates on $C_{0}$ in the sense of least squares to a given function $F(z)$ defined on $C_{0}$; in Problem 3 the function $f_{M}(z)$ approximates on $S$ in the sense of least squares to a given function $F(z)$ defined on $S$; in Problem 4 the set $S$ consists of disjoint Jordan regions, in each of which there is a single condition of interpolation, and $f_{M}(z)$ approximates on $C_{0}$ in the sense of least pth powers to a function $F(z)$ defined on $C_{0}$. We shall consider also analogous problems in which the norm $M$ of $f_{M}(z)$ in $R$ is an integral over $C_{1}$ instead of an upper bound $M$ of the modulus.

The existence of the functions $f_{M}(z)$ of best approximation is easily proved in every case [cf. 8, pp. 477-478], and is left to the reader. The uniqueness of the $f_{M}(z)$ follows easily in certain cases [as in 12, p. 243] but is not necessary for our present purposes.

Received by the editors February 13, 1950.

(1) Numbers in brackets refer to the references cited at the end of the paper.

(2) If a letter denotes an open set, the letter with a dash over it denotes the closure of that set. 
In the detailed study of convergence of the functions $f_{M}(z)$ we shall need further notation. We denote by $\phi(z)$ the function harmonic in $R-\bar{S}$, continuous on $\bar{R}-S$, equal to zero and unity on $C_{0}$ and $C_{1}$ respectively; by $C_{\nu}, 0 \leqq \nu \leqq 1$, we denote generically the locus $\phi(z)=\nu$; by $R_{\nu}$ the point set consisting of $\bar{S}$ together with the points of $R-\bar{S}$ for which $0<\phi(z)<\nu$, by $\bar{R}_{\nu}$ the corresponding closed set.

2. Preliminary results. For reference we state two theorems $[10, \mathrm{pp}$. 294,295 ] of application to several proofs in this paper.

THEOREM 2.1. Let $D$ be a region whose boundary $B$ consists of a finite number of Jordan arcs $J_{k}$, mutually disjoint except possibly at end points $E_{j}$. Let the function $V(z)$ be harmonic and bounded in $D$, continuous in the corresponding closed region $\bar{D}$ except in the points $E_{j}$, constant on each $J_{k}$.

Let the functions $F_{n}(z), n=1,2, \cdots$, be uniformly bounded in $D$, locally analytic except perhaps for branch points although not necessarily single-valued in $D$, but let $\left|F_{n}(z)\right|$ be single-valued in $D$ and continuous in $\bar{D}$ except perhaps in the points $E_{j}$; and suppose we have for each $k$

$$
\limsup _{n \rightarrow \infty}\left[1 . \text { u.b. }\left|F_{n}(z)\right|, z \text { on } J_{k}\right] \leqq\left[e^{V(z)}, \text { z on } J_{k}\right] \text {. }
$$

Then on every closed set $Q$ in $\bar{D}$ containing no point $E_{j}$ we have

$$
\limsup _{n \rightarrow \infty}\left[\max \left|F_{n}(z)\right|, z \text { on } Q\right] \leqq\left[\max e^{V(z)}, z \text { on } Q\right] \text {. }
$$

THEOREM 2.2. Under the conditions of Theorem 2.1 suppose the equality sign holds in (2.1) for a particular continuum $Q_{0}$ in $D\left(Q_{0}\right.$ may be a single point):

$$
\underset{n \rightarrow \infty}{\limsup }\left[\max \left|F_{n}(z)\right|, z \text { on } Q_{0}\right]=\left[\max e^{V(z)}, z \text { on } Q_{0}\right] \text {. }
$$

Then on every continuum $Q$ in $\bar{D}$ consisting of more than one point and containing no point $E_{j}$ we have the corresponding equality:

$$
\underset{n \rightarrow \infty}{\limsup }\left[\max \left|F_{n}(z)\right|, z \text { on } Q\right]=\left[\max e^{V(z)}, z \text { on } Q\right] \text {. }
$$

We use Theorems 2.1 and 2.2 in the proof of

LeMмA 2.1. If $f(z)$ is a function analytic throughout $R_{\rho}, 0<\rho<1$, but coincides on $\bar{S}$ with no function analytic throughout any $R_{\rho^{\prime}}, \rho^{\prime}>\rho$, if $\left\{F_{n}(z)\right\}$ is a sequence of functions analytic in $R$ and satisfying on every closed subset $\bar{R}^{\prime}$ of $R$ the condition

$$
\limsup _{n \rightarrow \infty}\left[\max \left|F_{n}(z)\right|, z \text { in } \bar{R}^{\prime}\right]^{1 / n} \leqq e^{\alpha}, \quad \alpha>0,
$$

and on every closed subset $\bar{S}_{1}$ of $S$ the condition 
then we have $\alpha \rho+\beta-\beta \rho \geqq 0$; moreover we have whenever $0 \leqq \sigma<\rho$,

$$
\limsup _{n \rightarrow \infty}\left[\max \left|f(z)-F_{n}(z)\right|, z \text { in } \bar{R}_{\sigma}\right]^{1 / n} \leqq e^{\alpha \sigma+\beta-\beta \sigma},
$$

and whenever $\rho \leqq \sigma<1$,

$$
\limsup _{n \rightarrow \infty}\left[\max \left|F_{n}(z)\right|, z \text { in } \bar{R}_{\sigma}\right]^{1 / n} \leqq e^{\alpha \sigma+\beta-\beta \sigma} .
$$

If $\alpha \rho+\beta-\beta \rho=0$ we have whenever $0 \leqq \sigma<\rho$,

$$
\limsup _{n \rightarrow \infty}\left[\max \left|f(z)-F_{n}(z)\right|, z \text { in } \bar{R}_{\sigma}\right]^{1 / n}=e^{(\sigma-\rho)(\alpha-\beta)},
$$

and whenever $\rho \leqq \sigma<1$,

$$
\limsup _{n \rightarrow \infty}\left[\max \left|F_{n}(z)\right|, z \text { in } \bar{R}_{\sigma}\right]^{1 / n}=e^{(\sigma-\rho)(\alpha-\beta)} .
$$

Let $C_{0}$ consist of $s$ Jordan curves, and for each $k$ let $\Gamma_{0}^{(k)}$ consist of $s$ disjoint Jordan curves in $S$ geometrically near the respective curves of $C_{0}$, and let the sequence $\Gamma_{0}^{(1)}, \Gamma_{0}^{(2)}, \cdots$ approach $C_{0}$ monotonically. Let $C_{1}$ consist of $t$ Jordan curves, and for each $k$ let $\Gamma_{1}^{(k)}$ consist of $t$ disjoint Jordan curves exterior to $R$ near the respective curves of $C_{1}$, and let the sequence $\Gamma_{1}^{(k)}$ approach $C_{1}$ monotonically. Denote by $V_{k}(z)$ the function harmonic in the region $T^{(k)}$ bounded by $\Gamma_{0}^{(k)}$ and $\Gamma_{1}^{(k)}$, continuous in $\bar{T}^{(k)}$, zero on $\Gamma_{0}^{(k)}$, and unity on $\Gamma_{1}^{(k)}$. In $T^{(k)}$ let $\Gamma_{\nu}^{(k)}$ denote generically the locus $V_{k}(z)=\nu$. As $k$ becomes infinite, the functions $V_{k}(z)$ approach uniformly in $\bar{R}-S$ the function $\phi(z)$ [Lebesgue, 3]. Denote by $S^{(k)}$ the subset of $S$ bounded by $\Gamma_{0}^{(k)}$ and by $R^{(k)}$ the point set containing $R$ bounded by $\Gamma_{1}^{(k)}$. Denote by $R_{\nu}^{(k)}$ the set $\bar{S}^{(k)}$ plus the set of $T^{(k)}$ on which we have $0<V_{k}(z)<\nu$. For a given $\eta, \rho>\eta>0$, and for $k$ sufficiently large, the locus $C_{0}$ lies in $R_{\eta}^{(k)}$, the locus $C_{1}$ lies exterior to $R_{1-\eta}^{(k)}$ and the locus $C_{\rho}$ lies in $R_{\rho+\eta}^{(k)}$. From our hypothesis, for a given $\eta, \rho>\eta>0$, and for $k$ sufficiently large, we have for arbitrary $\epsilon>0$, and $n$ sufficiently large,

$$
\left|F_{n+1}(z)-F_{n}(z)\right| \leqq e^{n(\alpha+e)}, \quad z \text { on } \Gamma_{1-\eta}^{(k)}
$$

and

$$
\left|F_{n+1}(z)-F_{n}(z)\right| \leqq e^{n(\beta+\epsilon)}, \quad z \text { on } \Gamma_{0}^{(k)} \text {. }
$$

We may now apply Theorem 2.1, setting $F_{n}(z)$ of the theorem equal to $\left[F_{n+1}(z)-F_{n}(z)\right]^{1 / n}$ and $V(z)$ equal to $\beta+(\alpha-\beta) V_{k}(z) /(1-\eta)$, to obtain for $0<\sigma+\eta<1-\eta$,

$$
\underset{n \rightarrow \infty}{\limsup }\left[\max \left|F_{n+1}(z)-F_{n}(z)\right|, z \text { in } \bar{R}_{\sigma+\eta}^{(k)}\right]^{1 / n} \leqq e^{\beta+(\alpha-\beta)(\sigma+\eta) /(1-\eta)} .
$$


For $k$ sufficiently large, $\bar{R}_{\sigma+\eta}^{(k)}$ contains $\bar{R}_{\sigma}$ in its interior. If we now allow $\eta$ to approach zero we have

$$
\limsup _{n \rightarrow \infty}\left[\max \left|F_{n+1}(z)-F_{n}(z)\right|, z \text { in } \bar{R}_{\sigma}\right]^{1 / n} \leqq e^{\alpha \sigma+\beta-\beta \sigma}, \quad 0 \leqq \sigma<1 .
$$

If $\alpha \rho+\beta-\beta \rho$ is negative, then for suitably chosen $\rho^{\prime}, 1>\rho^{\prime}>\rho, \alpha \rho^{\prime}+\beta-\beta \rho^{\prime}$ is negative, and the sequence $\left\{F_{n}(z)\right\}$ converges uniformly in the closed region $\bar{R}_{\rho^{\prime}}$. But the sequence converges uniformly to $f(z)$ in $\bar{S}_{1}$, and by hypothesis $f(z)$ is not analytic throughout $R_{\rho^{\prime}}, \rho^{\prime}>\rho$. This contradiction leads to the conclusion $\alpha \rho+\beta-\beta \rho \geqq 0$.

For arbitrary $\epsilon>0$, for $n$ sufficiently large, and for $0 \leqq \sigma<1$, the inequality

$$
\left|F_{n+1}(z)-F_{n}(z)\right| \leqq e^{(\alpha \sigma+\beta-\beta \sigma+\epsilon) n}
$$

is valid in $\bar{R}_{\sigma}$. From this inequality and from the two equations

$$
\begin{aligned}
& f(z) \equiv F_{n}(z)+\left[F_{n+1}(z)-F_{n}(z)\right]+\left[F_{n+2}(z)-F_{n+1}(z)\right]+\cdots, \quad 0 \leqq \sigma<\rho, \\
& F_{n+1}(z) \equiv F_{N}(z)+\left[F_{N+1}(z)-F_{N}(z)\right]+\left[F_{N+2}(z)-F_{N+1}(z)\right]+\cdots \\
& +\left[F_{n+1}(z)-F_{n}(z)\right] \text {, } \\
& n \geqq N \text {, }
\end{aligned}
$$

follow at once the inequalities

$$
\begin{array}{ll}
\limsup _{n \rightarrow \infty}\left[\max \left|f(z)-F_{n}(z)\right|, z \text { in } \bar{R}_{\sigma}\right]^{1 / n} \leqq e^{\alpha \sigma+\beta-\beta \sigma}, & 0 \leqq \sigma<\rho, \\
\limsup _{n \rightarrow \infty}\left[\max \left|F_{n}(z)\right|, z \text { in } \bar{R}_{\sigma}\right]^{1 / n} \leqq e^{\alpha \sigma+\beta-\beta \sigma}, & \rho \leqq \sigma<1 .
\end{array}
$$

If $\alpha \rho+\beta-\beta \rho=0$, we show that the equality signs must hold in (2.3) and (2.4). We first prove the equality valid for $z$ on $C_{\rho}$. We assume the strong inequality holds in (2.2) and show this assumption leads to a contradiction. Under the assumption we have

$$
\underset{n \rightarrow \infty}{\lim \sup }\left[\max \left|F_{n+1}(z)-F_{n}(z)\right|, z \text { on } C_{p}\right]^{1 / n} \leqq e^{\gamma}<1 .
$$

We again apply Theorem 2.1 , this time to the region bounded by $C_{\rho}$ and $C_{1-\eta}$, $1-\eta>\rho$, and we set $V(z)=[\gamma(1-\eta)-\alpha \rho+(\alpha-\gamma) \phi(z)] /(1-\rho-\eta)$. We obtain for $\rho<\sigma<1-\eta$

$$
\underset{n \rightarrow \infty}{\lim \sup }\left[\max \left|F_{n+1}(z)-F_{n}(z)\right|, z \text { in } \bar{R}_{\sigma}\right]^{1 / n} \leqq e^{[\gamma(1-\eta)-\alpha \rho+(\alpha-\gamma) \sigma] /(1-\rho-\eta)} .
$$

From this inequality we infer at once that the sequence $\left\{F_{n}(z)\right\}$ converges uniformly interior to some $C_{\rho^{\prime}}, \rho^{\prime}>\rho$, and we have just shown such convergence impossible.

From this proof that the equality sign holds in (2.2) on $C_{\rho}$ we are now able to conclude that the equality signs must hold in (2.3) and (2.4), for Theorem 2.2 shows 
$\lim \sup \left[\max \left|F_{n+1}(z)-F_{n}(z)\right|, z \text { in } \bar{R}_{\sigma}\right]^{1 / n}=e^{(\alpha-\beta)(\sigma-\rho)}, \quad 0 \leqq \sigma<1 ;$

and elementary inequalities show that the inequality signs in (2.3) and (2.4) would contradict this equation.

A closely related lemma which we shall later use is

LEMMA 2.2. Let $f(z)$ be the function defined in Lemma 2.1, and let $f_{M}(z)$ be a set of functions analytic in $R$ satisfying on every closed subset $\bar{R}^{\prime}$ of $R$ the condition

$$
\limsup _{M \rightarrow \infty}\left[\max \left|f_{M}(z)\right|, z \text { in } \bar{R}^{\prime}\right]^{1 / \log M} \leqq e^{\alpha}, \quad \alpha>0,
$$

and on every closed subset $\bar{S}_{1}$ of $S$ the condition

$$
\limsup _{M \rightarrow \infty}\left[\max \left|f(z)-f_{M}(z)\right|, z \text { in } \bar{S}_{1}\right]^{1 / \log M} \leqq e^{\beta}, \quad \beta<0 .
$$

If $\alpha \rho+\beta-\beta \rho=0$, we have, whenever $0 \leqq \sigma<\rho$,

$$
\underset{M \rightarrow \infty}{\limsup }\left[\max \left|f(z)-f_{M}(z)\right|, z \text { in } \bar{R}_{\sigma}\right]^{1 / \log M}=e^{(\sigma-\rho)(\alpha-\beta)},
$$

and whenever $\rho \leqq \sigma<1$,

$$
\limsup _{M \rightarrow \infty}\left[\max \left|f_{M}(z)\right|, z \text { in } \bar{R}_{\sigma}\right]^{1 / \log M}=e^{(\sigma-\rho)(\alpha-\beta)} .
$$

The proof of Lemma 2.2 is essentially the same as the proofs of lemmas [4, pp. 58-61] dealing with the analogous situation where $R$ and $\bar{S}$ replace $\bar{R}^{\prime}$ and $\bar{S}_{1}$ respectively.

3. Problem 1. The simplest case that concerns us is that of interpolation to the given function, assumed analytic on $\bar{S}$ :

LEMMA 3.1. Let the function $f(z)$ analytic in $R$ approximate to a function $F(z)$ in $S:|f(z)-F(z)|<\epsilon$ in $S$. Let the points $z_{k}, k=1,2, \cdots, \lambda$, not necessarily distinct $\left({ }^{3}\right)$, lie in $S$. Then there exists a function $f_{0}(z)$ analytic in $R$ which interpolates to $F(z)$ in $z_{k}$ such that in $S$ we have $\left|f_{0}(z)-F(z)\right|<(K+1) \epsilon$, and in $R$ we have $\left|f_{0}(z)\right|<K \epsilon+1 . u . b .[|f(z)|, z$ in $R]$, where $K$ is independent of $\epsilon$.

There exists a polynomial $p(z)$ which takes on the values $\left[f\left(z_{k}\right)-F\left(z_{k}\right)\right]$ in the points $z_{k}$, and is such that $|p(z)|<K \epsilon$ in $R$, where $K$ is a suitably chosen constant $\left[7\right.$, pp. 310-312] independent of $\epsilon$. The function $f_{0}(z) \equiv f(z)$ $-p(z)$ is analytic in $R$, interpolates to $F(z)$ in $z_{k}$, and satisfies the required inequalities.

THEOREM 3.1. Let the function $f(z)$ be analytic throughout $R_{\rho}$, but not through-

(3) A condition of interpolation at assigned points $z_{k}$ which are not all distinct is considered to imply $[7$, p. 50] a condition on the derivatives of the interpolating functions at multiple points $z_{k}$. 
out any $R_{\rho^{\prime}}, \rho^{\prime}>\rho>0$. Let the points $z_{k}, k=1,2, \cdots, \lambda$, not necessarily distinct, lie in $S$. Let $g_{M}(z)$ be a function analytic and of modulus not greater than $M$ in $R$, coinciding with $f(z)$ in the points $z_{k}$, such that $\max \left[\left|f(z)-g_{M}(z)\right|, z\right.$ in $\left.\bar{S}\right]$ is least. Then we have $\lim _{M \rightarrow \infty} g_{M}(z)=f(z)$ throughout $R_{\rho}$, uniformly on any closed set in $R_{p}$; more explicitly, we have

$$
\begin{array}{ll}
\limsup _{M \rightarrow \infty}\left[\max \left|f(z)-g_{M}(z)\right|, z \text { in } \bar{R}_{\nu}\right]^{1 / \log M}=e^{(\nu-\rho) /(1-\rho)}, & 0 \leqq \nu<\rho, \\
\limsup _{M \rightarrow \infty}\left[\operatorname{l.u.b} .\left|g_{M}(z)\right|, z \text { in } R_{\nu}\right]^{1 / \log M}=e^{(\nu-\rho) /(1-\rho)}, & \rho \leqq \nu \leqq 1 .
\end{array}
$$

There exists $\left[4\right.$, p. 57] a function $g_{M}^{0}(z)$ analytic and of modulus not greater than $M$ in $R$ such that

$$
\limsup _{M \rightarrow \infty}\left[\max \left|f(z)-g_{M}^{0}(z)\right|, z \text { in } \bar{R}_{\nu}\right]^{1 / \log M}=e^{(\nu-\rho) /(1-\rho)}, \quad 0 \leqq \nu<\rho .
$$

We have thus for arbitrary $\epsilon(>0)$ and $M$ sufficiently large

$$
\max \left[\left|f(z)-g_{M}^{0}(z)\right|, z \text { in } \bar{S}\right] \leqq(M-\epsilon)^{-\rho /(1-\rho)} .
$$

Here we set $g_{M_{1}}^{\prime}(z) \equiv g_{M}^{0}(z)+p_{M}(z)$, where $p_{M}\left(z_{k}\right)=f\left(z_{k}\right)-g_{M}^{0}\left(z_{k}\right)$, and we have, by Lemma 3.1,

$$
\begin{gathered}
\max \left[\left|f(z)-g_{M_{1}}^{\prime}(z)\right|, z \text { in } \bar{S}\right] \leqq(K+1)(M-\epsilon)^{-\rho /(1-\rho)}, \\
\quad K \text { independent of } M \text { and } \epsilon, \\
\text { l.u.b. }\left[\left|g_{M_{1}}^{\prime}(z)\right|, z \text { in } R\right] \leqq M+K(M-\epsilon)^{-\rho /(1-\rho)}=M_{1} .
\end{gathered}
$$

From these inequalities we obtain

$\max \left[\left|f(z)-g_{M_{1}}^{\prime}(z)\right|, z\right.$ in $\left.\bar{S}\right] \leqq K^{\prime}\left(M_{1}-\epsilon\right)^{-\rho /(1-\rho)}, \quad K^{\prime}$ independent of $M_{1}$.

This last inequality holds a fortiori if $g_{M_{1}}^{\prime}(z)$ is replaced by $g_{M_{1}}(z)$; equations (3.1) and (3.2) now follow [4, pp. 58-59].

Equations (3.1) and (3.2) are more generally a consequence of (3.1) for $\nu=0$ and $\left|g_{M}(z)\right| \leqq M$ in $R$, whether or not $g_{M}(z)$ is the extremal function.

THEOREM 3.2. Let the point set $S$ consist of the respective interiors of mutually exterior Jordan curves $C_{0}^{(1)}, C_{0}^{(2)}, \cdots, C_{0}^{(s)}$. Let $F(z)$ be meromorphic in $S$, continuous on $C_{0}$ (in the two-dimensional sense, with respect to $\bar{S}$ ), and let the points $z_{k}, k=1,2, \cdots, \lambda$, (not necessarily distinct) in $S$ and functional values $A_{k}, k=1,2, \cdots, \lambda$, be given. Let $f(z)$ be the unique function analytic in $S$, continuous in $\bar{S}$, which takes on the values $A_{k}$ in the points $z_{k}$, and such that $\mu_{j}=\left[\max |f(z)-F(z)|, z\right.$ on $\left.C_{0}^{(j)}\right]$ is least.

Let $f_{M}(z)$ be (for sufficiently large $M$ ) the function analytic and of modulus not greater than $M$ in $R$, which satisfies $f_{M}\left(z_{k}\right)=A_{k}$, and for which $\sum_{j=1}^{s}\left[\max \mid f_{M}(z)\right.$ $-F(z) \mid$, z on $\left.C_{0}^{(j)}\right]$ is least. Then we have $\lim _{M \rightarrow \infty} f_{M}(z)=f(z)$ in $S$, uniformly 
on any closed set in $S$. If $f(z)$ is analytic on $\bar{S}$, let $\rho$ denote the largest number $(0<\rho<1)$ such that $f(z)$ is analytic throughout $R_{p}$. Then the analogues of equations (3.1) and (3.2) with $g_{M}(z)$ replaced by $f_{M}(z)$ are valid. whence

It is to be noticed that we have $\max \left[\left|f_{M}(z)-F(z)\right|, z\right.$ on $\left.C_{0}^{(j)}\right] \geqq \mu_{j}$, $\max \left[\left|f_{M}(z)-F(z)\right|, z\right.$ on $\left.C_{0}^{(j)}\right]-\mu_{j}$

$$
\leqq \sum_{j=1}^{s}\left\{\max \left[\left|f_{M}(z)-F(z)\right|, z \text { on } C_{0}^{(j)}\right]-\mu_{j}\right\} .
$$

In Theorem 3.2 the case $\lambda=0$ is not excluded, and means simply the omission of conditions of interpolation.

The existence of the function $f(z)$ and various of its properties are known [7, pp. 335-338], including the continuity of $f(z)$ in $\bar{S}$ as a consequence of the remaining requirements on $f(z)$. The modulus $|f(z)-F(z)|$ is constant on each component of $C_{0}$. The extremal function $f(z)$ is analytic on a component $C_{0}^{(j)}$ if the curve $C_{0}^{(j)}$ is analytic.

We prove the theorem for the case $s=1$; the extension to the more general case is immediate.

Since the function $f(z)$ is continuous in $\bar{S}$, there exists $[7$, p. 47 , Theorem 15] a sequence of rational functions $r_{k}(z)$ with no poles in $\bar{R}$ such that we have $\lim _{k \rightarrow \infty} r_{k}(z)=f(z)$ uniformly in $\bar{S}$. These functions may be chosen to coincide with $f(z)$ in the prescribed points $[7$, p. 313]. Thus we have

$$
\lim _{M \rightarrow \infty}\left[\max \left|f_{M}(z)-F(z)\right|, z \text { on } C_{0}\right]=\max \left[|f(z)-F(z)|, z \text { on } C_{0}\right]=\mu_{1} \text {. }
$$

The first part of Theorem 3.2 now follows at once $[7$, p. 336].

It has been proved $\left[7\right.$, p. 338] that if the $f_{n}(z)$ are functions analytic in $S$, if $M_{n}=1$. u.b. $\left[\left|f_{n}(z)-F(z)\right|, z\right.$ in $\left.S, z \rightarrow C_{0}\right]$, and if $\lim _{n \rightarrow \infty} M_{n}=\mu_{1}$, then the inequality

$$
\left|f_{n}(z)-f(z)\right| \leqq Q\left(M_{n}-\mu_{1}\right)
$$

is valid for $n$ sufficiently large, for $z$ on any closed set $\bar{S}_{1}$ interior to $S$, where $Q$ depends on $\bar{S}_{1}$ but not on $n$. For the functions $g_{M}(z)$ analytic and of modulus not greater than $M$ in $R$, coinciding with $f(z)$ in the points $z_{k}$, such that $\max \left[\left|f(z)-g_{M}(z)\right|, z\right.$ in $\left.\bar{S}\right]$ is least, we have by Theorem 3.1

$$
\limsup _{M \rightarrow \infty}\left[\max \left|f(z)-g_{M}(z)\right|, z \text { on } C_{0}\right]^{1 / \log M}=e^{-\rho /(1-\rho)} \text {. }
$$

Thus we have for $z$ on $C_{0}$, for arbitrary $\epsilon>0$, and for $M$ sufficiently large,

$$
\left|g_{M}(z)-F(z)\right| \leqq e^{(-\rho+\epsilon) \log M /(1-\rho)}+\max \left[|f(z)-F(z)|, z \text { on } C_{0}\right] ;
$$

this inequality must certainly remain valid if we replace the functions $g_{M}(z)$ 
by the functions $f_{M}(z)$ of Theorem 3.2:

$$
\max \left[\left|f_{M}(z)-F(z)\right|, z \text { on } C_{0}\right] \leqq e^{(-\rho+e) \log M /(1-\rho)}+\mu_{1} ;
$$

it follows by inequality (3.3) that for $\epsilon>0$ and $M$ sufficiently large,

$$
\left|f_{M}(z)-f(z)\right| \leqq Q e^{(-\rho+\epsilon) \log M /(1-\rho)}, \quad z \text { in } \bar{S}_{1}, Q \text { independent of } M \text {. }
$$

The functions $f_{M}(z)$ satisfy the conditions of Lemma 2.2, with $\alpha=1$, and the second part of Theorem 3.2 now follows from the lemma.

The conclusion of Theorem 3.2 may apply even if the $f_{M}(z)$ are not extremal functions:

COROLlary 1. Under the conditions of Theorem 3.2, let $\psi_{M}(z)$ be analytic and of modulus not greater than $M$ in $R$, and satisfy the auxiliary conditions with

$$
\lim _{M \rightarrow \infty}\left\{\sum_{j=1}^{s}\left[\max \left|\psi_{M}(z)-F(z)\right|, z \text { on } C_{0}^{(j)}\right]\right\}=\sum_{j=1}^{s} \mu_{j} \text {. }
$$

Then the set of functions $\psi_{M}(z)$ converges to $f(z)$ throughout $S$, uniformly on any closed set interior to $S$. If $f(z)$ is analytic throughout $R_{\rho}$ but not throughout any $R_{\rho^{\prime}}, \rho^{\prime}>\rho>0$, and if we also have

$$
\limsup _{M \rightarrow \infty}\left\{\sum_{j=1}^{s}\left[\max \left|\psi_{M}(z)-F(z)\right|, z \text { on } C_{0}^{(j)}-\mu_{j}\right]\right\}^{1 / \log M} \leqq e^{-\rho /(1-\rho)},
$$

then the analogues of (3.1) and (3.2) with $g_{M}(z)$ replaced by $\psi_{M}(z)$ are valid.

Conclusions analogous to those of Theorem 3.2 may still follow when the norm of $f_{M}(z)$ in $R$ is no longer an upper bound of the modulus, as the reader may establish:

Corollary 2. Let $q(>0)$ be given. Let the hypothesis of Theorem 3.2 be modified so that the inequality $\left|f_{M}(z)\right| \leqq M$ in $R$ is replaced by $\int_{C_{\nu}}\left|f_{M}(z)\right| q|d z|$ $\leqq M^{q}$, for all $\nu, 0<\nu<1$. Then we have $\lim _{M \rightarrow \infty} f_{M}(z)=f(z)$ in $S$, uniformly on any closed set in $S$. If $f(z)$ is analytic throughout $R_{\rho}$ but not throughout any $R_{\rho^{\prime}}$, $\rho^{\prime}>\rho>0$, we have

$$
\begin{array}{ll}
\limsup _{M \rightarrow \infty}\left[\max \left|f(z)-f_{M}(z)\right|, z \text { in } \bar{R}_{\nu}\right]^{1 / \log M}=e^{(\nu-\rho) /(1-\rho)}, & 0 \leqq \nu<\rho, \\
\left.\limsup _{M \rightarrow \infty} \text { [l.u.b. }\left|f_{M}(z)\right|, z \text { in } R_{\nu}\right]^{1 / \log M}=e^{(\nu-\rho) /(1-\rho)}, & \rho \leqq \nu \leqq 1 .
\end{array}
$$

An analogue of Corollary 1 involving non-extremal functions is also true.

4. Problem 2. Let $C$ be a rectifiable Jordan curve, and let prescribed conditions of interpolation $f\left(z_{k}\right)=A_{k}(k=1,2, \cdots, \lambda)$ be given interior to $C$. Any function $f(z)$ analytic interior to $C$, continuous in the closed interior $\bar{C}$ of $C$, and satisfying the conditions of interpolation can be uniformly approximated in $\bar{C}$ by a polynomial which satisfies the conditions of interpolation 
[7, p. 310]. If the polynomial $p_{1}(z)$ satisfies the conditions of interpolation and if we set $p_{0}(z) \equiv\left(z-z_{1}\right)\left(z-z_{2}\right) \cdots\left(z-z_{\lambda}\right)$, the function $f(z)$ can be uniformly approximated in $\bar{C}$ (as closely as desired) by $p_{1}(z)$ plus a linear combination of a finite number of the functions $p_{0}(z), z p_{0}(z), z^{2} p_{0}(z), \cdots$ We denote by $(B)$ the class of functions on $C$ each consisting of $p_{1}(z)$ plus a function of the closed extension $[7, p .123]$ on $C$ of the class $p_{0}(z), z p_{0}(z)$, $z^{2} p_{0}(z), \cdots$ Thus $[7, \S 11.5]$ a function of class $B$ is of class $L^{2}$ on $C$, and the Cauchy integral of such a function over $C$ is analytic interior to $C$, and satisfies the given conditions of interpolation. The class $B$ is independent of the particular $p_{1}(z)$ chosen.

An arbitrary function $F(z)$ of class $L^{2}$ on $C$ possesses a formal expansion consisting of $p_{1}(z)$ plus the formal expansion of $F(z)-p_{1}(z)$ in the orthogonalization on $C$ of the set $p_{0}(z), z p_{0}(z), z^{2} p_{0}(z), \cdots$; this formal expansion of $F(z)$ converges in the mean on $C$, and the limit in the mean is the function $f(z)$ of class $(J)$ of best approximation to $F(z)$ on $C$ in the sense of least squares. The Cauchy integral of $f(z)$ over $C$ represents interior to $C$ a function, also denoted by $f(z)$, analytic interior to $C$ and satisfying the auxiliary conditions. The formal expansion of $F(z)$ converges to $f(z)$ throughout the interior of $C$, uniformly on any closed set interior to $C$. If $C$ is sufficiently smooth, a specific formula for the function $f(z)$ is readily obtained $[7, \S 11.5]$ by a conformal map; if $C$ is an analytic Jordan curve and if $F(z)$ is analytic on $C$, then $f(z)$ is analytic in the closed interior of $C$.

The class $(5)$ thus defined for the interior of a rectifiable Jordan curve $C$ can also be defined for a bounded multiply connected region $S$ whose boundary $C$ consists of a finite number of disjoint rectifiable Jordan curves. Again we assume prescribed conditions of interpolation $f\left(z_{k}\right)=A_{k}(k=1,2, \cdots, \lambda)$ at points of $S$. Choose points $\beta_{1}, \beta_{2}, \cdots, \beta_{\gamma}$, precisely one in each of the regions into which $S+C$ separates the plane, and let $q_{0}(z), q_{1}(z), \cdots$ be an ordering of the functions $1,\left(z-\beta_{1}\right)^{-1},\left(z-\beta_{1}\right)^{-2}, \cdots,\left(z-\beta_{2}\right)^{-1},\left(z-\beta_{2}\right)^{-2}, \cdots, \cdots$, $\left(z-\beta_{\gamma}\right)^{-1},\left(z-\beta_{\gamma}\right)^{-2}, \cdots ;$ in the case $\beta_{j}=\infty$ we replace $\left(z-\beta_{j}\right)^{-n}$ here and subsequently by $z^{n}$. Let the polynomial $p_{1}(z)$ satisfy the conditions of interpolation and set $p_{0}(z) \equiv\left(z-z_{1}\right)\left(z-z_{2}\right) \cdots\left(z-z_{\lambda}\right)$ as before. If the function $f(z)$ is analytic in $S$, continuous in $S+C$, and satisfies the conditions of interpolation, then $[7$, p. 313] the function $f(z)$ can be uniformly approximated in $S+C$ by a rational function which satisfies the conditions of interpolation and has its poles in the points $\beta_{j}$. Thus $f(z)$ can be uniformly approximated in $S+C$ by $p_{1}(z)$ plus a linear combination of a finite number of the functions $p_{0}(z) q_{0}(z)$, $p_{0}(z) q_{1}(z), p_{0}(z) q_{2}(z), \cdots$. We denote by (s) the class of functions on $C$ each consisting of $p_{1}(z)$ plus a function of the closed extension [7, p. 123] on $C$ of the class $p_{0} q_{0}, p_{0} q_{1}, p_{0} q_{2}, \cdots$. Then a function of class $B$ is of class $L^{2}$ on $C$, and the Cauchy integral of such a function over $C$ is analytic in $S$ and satisfies the given conditions of interpolation. An arbitrary function $F(z)$ of class $L^{2}$ on $C$ possesses a formal development consisting of $p_{1}(z)$ plus the formal expansion 
of $F(z)-p_{1}(z)$ in the orthogonalization of the set $p_{0} q_{0}, p_{0} q_{1}, p_{0} q_{2}, \cdots$; this formal expansion of $F(z)$ converges in the mean on $C$, and the limit in the mean is the function $f(z)$ of class $B$ of best approximation to $F(z)$ on $C$ in the sense of least squares; the Cauchy integral of $f(z)$ over $C$ represents in $S$ a function, denoted by $f(z)$, analytic in $S$ and satisfying the auxiliary conditions. The formal expansion of $F(z)$ converges to $f(z)$ throughout $S$, uniformly on any closed set in $S$.

When we envisage simultaneously several regions $S$, the class \&5 for their total boundary consists of all functions belonging to the class $B$ for each of the regions.

Let $S$ be a multiply connected region whose boundary $C$ consists of the disjoint rectifiable Jordan curves $C^{(1)}, C^{(2)}, \cdots, C^{(s)}$. The region $S$ lies in regions $S^{(1)}, S^{(2)}, \cdots, S^{(s)}$ bounded respectively by the curves $C^{(1)}, C^{(2)}, \cdots$, $C^{(s)}$. Any sequence of functions analytic in $\bar{S}$ convergent in the mean on $C$ is the sum of $s$ sequences of functions analytic respectively in the closed regions $S^{(j)}+C^{(j)}$ and converging in the mean on $C^{(j)}$. Each function of the class $\&$ for $S$ (or for $C$ ) is the sum of $s$ components, namely, functions of the respective classes $(\$)$ for the regions $S^{(j)}$, where no auxiliary conditions are assigned; the latter classes are readily investigated $[7, \S 11.5]$ by use of conformal mapping, if the curves $C^{(j)}$ are sufficiently smooth.

We return to our usual notation $(\$ 1)$ :

THEOREM 4.1. Let $C_{0}$ be rectifiable, let auxiliary conditions of interpolation $f\left(z_{k}\right)=A_{k}, k=1,2, \cdots, \lambda$, be given at points of $S$, and let \& be the corresponding class of functions defined on $C_{0}$. Let $F(z)$ be given of class $L^{2}$ on $C_{0}$ and let $f(z)$ be the function of class $B$ on $C_{0}$ such that

$$
\mu=\int_{C_{0}}|f(z)-F(z)|^{2}|d z|
$$

is least. Let $f_{M}(z)$ be the function analytic and of modulus not greater than $M$ in $R$ satisfying the auxiliary conditions such that

$$
\int_{C_{0}}\left|f_{M}(z)-F(z)\right|^{2}|d z|
$$

is least. Then the set $f_{M}(z)$ converges to $f(z)$ throughout $S$, uniformly on any closed subset of $S$. If the function $f(z)$ is analytic throughout $R_{\rho}$ but not throughout any $R_{\rho^{\prime}}, \rho^{\prime}>\rho>0$, then we have

$$
\begin{array}{ll}
\limsup _{M \rightarrow \infty}\left[\max \left|f(z)-f_{M}(z)\right|, z \text { in } \bar{R}_{\nu}\right]^{1 / \log M}=e^{(\nu-\rho) /(1-\rho)}, & 0 \leqq \nu<\rho, \\
\left.\lim _{M \rightarrow \infty} \text { [l.u.b. }\left|f_{M}(z)\right|, z \text { in } R_{\nu}\right]^{1 / \log M}=e^{(\nu-\rho) /(1-\rho)}, & \rho \leqq \nu \leqq 1 .
\end{array}
$$

We prove Theorem 4.1 for the case that $S$ consists of a single region, and leave the more general situation to the reader. 
The class $(B)$ on $C_{0}$ has been defined in terms of rational functions with poles in the points $\beta_{1}, \beta_{2}, \cdots, \beta_{\gamma}$, precisely one in each of the regions into which $S+C_{0}$ separates the plane. It is to be noticed that $B$ is independent of the particular choice of those points $\beta_{j}$; for of two classes of rational functions defined respectively in terms of two sets of distinct poles $\beta_{j}$, each rational function of the one class can be uniformly approximated in $S+C_{0}$ by a rational function of the other class, all rational functions satisfying the conditions of interpolation. In particular the points $\beta_{j}$ may be chosen exterior to $R[7, \mathrm{pp} .15,313]$; here we make use of the hypothesis that $\bar{S}$ separates no point of $R-\bar{S}$ from $C_{1}$.

The class (S) has been defined in terms of approximation on $C_{0}$ by interpolating rational functions satisfying the auxiliary conditions rather than by interpolating functions analytic and bounded in $R$, and satisfying the auxiliary conditions. However, each rational function considered is analytic and bounded in $R$; conversely, each function analytic and bounded in $R$ and satisfying the auxiliary conditions can be uniformly approximated in $\bar{R}_{\nu}$, $0<\nu<1$, by a rational function satisfying the auxiliary conditions; thus the two classes of approximating functions have the same set of limit functions, either continuous in $S+C_{0}$ and uniform limits, or of class $L^{2}$ on $C_{0}$, limits in the mean on $C_{0}$, and uniform limits on any closed set in $S$. The first part of Theorem 4.1 follows; we proceed to the second part.

Let $\mathrm{S}_{0}$ denote the closed extension on $C_{0}$ of the class $p_{0} q_{0}, p_{0} q_{1}, \cdots$. The formal expansion of the function $F(z)-p_{1}(z)$ in terms of the functions of class $\$_{0}$ converges in the mean on $C_{0}$ to the function $f(z)-p_{1}(z)$, and for any function $g(z)$ of class $\mathfrak{S}_{0}$ we have $[7$, p. 124]

$$
\int_{C_{0}}[F(z)-f(z)] \bar{g}(z)|d z|=0 .
$$

But the function $g_{M}(z)$ of Theorem 3.1 is of class (5), and $f(z)-g_{M}(z)$ is of class $\mathfrak{B S}_{0}$, so we have

$$
\begin{aligned}
\int_{C_{0}}\left|F(z)-g_{M}(z)\right|^{2}|d z|= & \int_{C_{\theta}}\left|F(z)-f(z)+f(z)-g_{M}(z)\right|^{2}|d z| \\
= & \int_{C_{0}}|F(z)-f(z)|^{2}|d z| \\
& +\int_{C_{0}}\left|f(z)-g_{M}(z)\right|^{2}|d z| .
\end{aligned}
$$

For $\epsilon>0$ and $M$ sufficiently large we therefore have

$$
\int_{C_{0}}\left|F(z)-g_{M}(z)\right|^{2}|d z| \leqq \mu+e^{2(-\rho+\epsilon) \log M /(1-\rho)} ;
$$


this inequality remains valid if we replace $g_{M}(z)$ by the function $f_{M}(z)$ of Theorem 4.1. The function $f(z)-f_{M}(z)$ is of the class $\$_{0}$, hence orthogonal on $C_{0}$ to $F(z)-f(z)$, so we have also

$$
\begin{aligned}
\int_{C_{0}}\left|F(z)-f_{M}(z)\right|^{2}|d z|= & \int_{C_{0}}|F(z)-f(z)|^{2}|d z| \\
& +\int_{C_{0}}\left|f(z)-f_{M}(z)\right|^{2}|d z|
\end{aligned}
$$

and, finally, for $\epsilon>0$ and $M$ sufficiently large,

$$
\int_{C_{0}}\left|f(z)-f_{M}(z)\right|^{2}|d z| \leqq e^{2(-\rho+e) \log M /(1-\rho)} .
$$

By means of the Cauchy integral formula and the Schwarz inequality we obtain

$$
\limsup _{M \rightarrow \infty}\left[\max \left|f(z)-f_{M}(z)\right|, z \text { in } \bar{S}_{1}\right]^{1 / \log M} \leqq e^{-\rho /(1-\rho)}
$$

Since we also have

$$
\limsup _{M \rightarrow \infty}\left[\max \left|f_{M}(z)\right|, z \text { in } \bar{R}^{\prime}\right]^{1 / \log M} \leqq e,
$$

Lemma 2.2 yields at once the conclusion of Theorem 4.1 .

COROLLARY 1. Under the conditions of Theorem 4.1, let $\psi_{M}(z)$ be analytic and of modulus not greater than $M$ in $R$, and satisfy the auxiliary conditions with

$$
\lim _{M \rightarrow \infty} \int_{C_{0}}\left|\psi_{M}(z)-F(z)\right| 2|d z|=\mu .
$$

Then the set of functions $\psi_{M}(z)$ converges to $f(z)$ throughout $S$, uniformly on any closed subset of $S$. If $f(z)$ is analytic throughout $R_{\rho}$ but not throughout any $R_{\rho^{\prime}}, \rho^{\prime}>\rho>0$, and if we also have

$$
\limsup _{M \rightarrow \infty}\left[\int_{C_{0}}\left|\psi_{M}(z)-F(z)\right|^{2}|d z|-\mu\right]^{1 / \log M} \leqq e^{-2 \rho /(1-\rho)},
$$

then the analogues of (4.1) and (4.2) with $f_{M}(z)$ replaced by $\psi_{M}(z)$ are valid.

Corollary 2. Let $q>0$ be given. Let Theorem 4.1 be modified so that $f_{M}(z)$ analytic in $R$ is required to satisfy the inequality $\int_{c_{\nu}}\left|f_{M}(z)\right| q|d z| \leqq M^{q}$ for all $\nu, 0<\nu<1$, instead of the inequality $\left|f_{M}(z)\right| \leqq M$ in $R$. Then the set $f_{M}(z)$ converges to $f(z)$ throughout $S$, uniformly on any closed subset of $S$. If the function $f(z)$ is analytic throughout $R_{\rho}$ but not throughout any $R_{\rho^{\prime}}, \rho^{\prime}>\rho>0$, then we have for all $t(>0)$ 


$$
\limsup _{M \rightarrow \infty}\left[\int_{C_{\nu}}\left|f(z)-f_{M}(z)\right| t|d z|\right]^{1 / t \log M}=e^{(\nu-\rho) /(1-\rho)}, \quad 0 \leqq \nu<\rho ;
$$

and, for all $t(>0)$ if $\rho \leqq \nu<1$, and for $0<t \leqq q$ if $\nu=1$,

$$
\limsup _{M \rightarrow \infty}\left[\int_{C_{\nu}}\left|f_{M}(z)\right| t|d z|\right]^{1 / t \log M}=e^{(\nu-\rho) /(1-\rho)} .
$$

These equations are valid for $t=\infty$, with the understanding that for a function $g(z)$ analytic in $R$ the integral $\left[\int_{C_{\nu}}|g(z)| t|d z|\right]^{1 / t}$ shall be interpreted for $t=\infty$ as l.u.b. $\left[|g(z)|, z\right.$ on $\left.C_{\nu}\right]$.

An analogue of Corollary 1 involving non-extremal functions is also true.

5. Problem 3. We proceed to discuss the analogue of the situations of $\S 4$, but where in the measure of approximation line integrals over the boundary of an open set are replaced by surface integrals over the open set. Let $S$ be a region whose boundary $C$ consists of a finite number of disjoint Jordan curves, and let conditions of interpolation $f\left(z_{k}\right)=A_{k}(k=1,2, \cdots, \lambda)$ in points of $S$ be prescribed. Choose points $\beta_{1}, \beta_{2}, \cdots, \beta_{\gamma}$, precisely one in each of the regions into which $S+C$ separates the plane, and let $q_{0}(z), q_{1}(z), \cdots$ be an ordering of the functions $1,\left(z-\beta_{1}\right)^{-1},\left(z-\beta_{1}\right)^{-2}, \cdots,\left(z-\beta_{2}\right)^{-1},\left(z-\beta_{2}\right)^{-2}$, $\cdots, \cdots,\left(z-\beta_{\gamma}\right)^{-1},\left(z-\beta_{\gamma}\right)^{-2}, \cdots$. Let the polynomial $p_{1}(z)$ satisfy the conditions of interpolation and set $p_{0}(z) \equiv\left(z-z_{1}\right)\left(z-z_{2}\right) \cdots\left(z-z_{\lambda}\right)$. If the function $f(z)$ is analytic in $S$, continuous in $S+C$, and satisfies the conditions of interpolation, then [7, p. 313] $f(z)$ can be uniformly approximated in $S+C$ by a rational function which satisfies the conditions of interpolation and has its poles in the points $\beta_{j}$. Thus $f(z)$ can be uniformly approximated in $S+C$ by $p_{1}(z)$ plus a linear combination of a finite number of the functions $p_{0}(z) q_{0}(z), p_{0}(z) q_{1}(z), \cdots$. We denote by $\Omega_{0}$ the class of functions on $S$ which is the closed extension $\left[7\right.$, p. 123] of the class $p_{0} q_{0}, p_{0} q_{1}, \cdots$; a function of class $\Omega_{0}$ is [7, p. 109] analytic in $S$ and vanishes in the points $z_{k}$; and we denote by $\Omega$ the class of functions on $S$ each consisting of $p_{1}(z)$ plus a function of class $\Omega_{0}$. Then a function of class $\Omega$ is analytic and of class $L^{2}$ on $S$, and satisfies the given conditions of interpolation. An arbitrary function $F(z)$ of class $L^{2}$ on $S$ possesses a formal expansion consisting of $p_{1}(z)$ plus the formal expansion of $F(z)-p_{1}(z)$ in terms of the class $\Omega_{0}$; this formal expansion of $F(z)$ converges in the mean on $S$, uniformly on any closed subset of $S$, and the limit in the mean on $S$ is the function $f(z)$ of class $\Omega$ of best approximation to $F(z)$ on $S$ in the sense of least squares.

If $S$ is simply connected and $C$ is sufficiently smooth, a specific formula for the function $f(z)$ is readily obtained $[7$, p. 328] by a conformal map; if $C$ is an analytic Jordan curve and $F(z)$ is analytic on $C$, then $f(z)$ is analytic in the closed interior of $C$.

When we envisage simultaneously several regions $S$, the class $\Omega$ for their 
sum consists of all functions belonging to the class $\Re$ for each of the regions.

THEOREM 5.1. Let auxiliary conditions of interpolation $f\left(z_{k}\right)=A_{k}$, $k=1,2, \cdots, \lambda$, be given at points of $S$, and let $\Omega$ be the corresponding class of functions defined on $S$. Let $F(z)$ be given of class $L^{2}$ on $S$ and let $f(z)$ be the function of class $\Re$ such that

$$
\mu=\iint_{S}|f(z)-F(z)|^{2} d S
$$

is least. Let $f_{M}(z)$ be the function analytic and of modulus not greater than $M$ in $R$ satisfying the auxiliary conditions such that

$$
\iint_{S}\left|f_{M}(z)-F(z)\right|^{2} d S
$$

is least. Then the set $f_{M}(z)$ converges to $f(z)$ throughout $S$, uniformly on any closed subset of $S$. If the function $f(z)$ is analytic throughout $R_{p}$ but not throughout any $R_{\rho^{\prime}}, \rho^{\prime}>\rho>0$, then we have

$$
\begin{array}{ll}
\limsup _{M \rightarrow \infty}\left[\max \left|f(z)-f_{M}(z)\right|, z \text { in } \bar{R}_{\nu}\right]^{1 / \log M}=e^{(\nu-\rho) /(1-\rho)}, & 0 \leqq \nu<\rho, \\
\limsup _{M \rightarrow \infty}\left[1 . \text { u.b. }\left|f_{M}(z)\right|, z \text { in } R_{\nu}\right]^{1 / \log M}=e^{(\nu-\rho) /(1-\rho)}, & \rho \leqq \nu \leqq 1 .
\end{array}
$$

We prove Theorem 5.1 only for the case that $S$ consists of a single region, and leave the more general situation to the reader. The first part of Theorem 5.1 follows as in $\$ 4$; we turn to the second part.

The formal expansion of the function $F(z)-p_{1}(z)$ in terms of the functions of class $\Omega_{0}$ converges in the mean on $S$ to the function $f(z)-p_{1}(z)$, and for any function $g(z)$ of class $\Omega_{0}$ we have

$$
\iint_{S}[F(z)-f(z)] \bar{g}(z) d S=0 .
$$

The function $g_{M}(z)$ of Theorem 3.1 is of class $\Re$, and $f(z)-g_{M}(z)$ is of class $\Omega_{0}$, so we have

$$
\iint_{S}\left|F(z)-g_{M}(z)\right|^{2} d S=\iint_{S}|F(z)-f(z)|^{2} d S+\iint_{S}\left|f(z)-g_{M}(z)\right|^{2} d S .
$$

For $\epsilon>0$ and $M$ sufficiently large we therefore have

$$
\iint_{S}\left|F(z)-g_{M}(z)\right|^{2} d S \leqq \mu+e^{2(-\rho+\epsilon) \log M /(1-\rho)} ;
$$

this inequality remains valid if we replace $g_{M}(z)$ by the function $f_{M}(z)$ of Theorem 5.1. The function $f(z)-f_{M}(z)$ is of class $\Omega_{0}$, hence orthogonal on $S$ 
to $F(z)-f(z)$, so we have also

$$
\iint_{S}\left|F(z)-f_{M}(z)\right|^{2} d S=\iint_{S}|F(z)-f(z)|^{2} d S+\iint_{S}\left|f(z)-f_{M}(z)\right|^{2} d S
$$

and, finally, for $\epsilon>0$ and $M$ sufficiently large,

$$
\iint_{S}\left|f(z)-f_{M}(z)\right|^{2} d S \leqq e^{2(-\rho+\epsilon) \log M /(1-\rho)} .
$$

From this inequality we obtain $[7$, p. 96]

$$
\limsup _{M \rightarrow \infty}\left[\max \left|f(z)-f_{M}(z)\right|, z \text { in } \bar{S}_{1}\right]^{1 / \log M} \leqq e^{-\rho /(1-\rho)} .
$$

Equations (5.1) and (5.2) now follow at once by the use of Lemma 2.2.

COROLlaRY 1. Under the conditions of Theorem 5.1, let $\psi_{M}(z)$ be analytic and of modulus not greater than $M$ in $R$, and satisfy the auxiliary conditions with

$$
\lim _{M \rightarrow \infty} \iint_{S}\left|\psi_{M}(z)-F(z)\right|^{2} d S=\mu .
$$

Then the set of functions $\psi_{M}(z)$ converges to $f(z)$ throughout $S$, uniformly on any closed subset of $S$. If $f(z)$ is analytic throughout $R_{\rho}$ but not throughout any $R_{\rho^{\prime}}, \rho^{\prime}>\rho>0$, and if we also have

$$
\limsup _{M \rightarrow \infty}\left[\iint_{S}\left|\psi_{M}(z)-F(z)\right|^{2} d S-\mu\right]^{1 / \log M} \leqq e^{-2 \rho /(1-\rho)},
$$

then the analogues of (5.1) and (5.2) with $f_{M}(z)$ replaced by $\psi_{M}(z)$ are valid.

Corollary 2. Let $q>0$ be given. Let Theorem 5.1 be modified so that $f_{M}(z)$ analytic in $R$ is required to satisfy the inequality $\iint_{R}\left|f_{M}(z)\right|{ }^{a} d S \leqq M^{a}$ instead of the inequality $\left|f_{M}(z)\right| \leqq M$ in $R$. Then the set $f_{M}(z)$ converges to $f(z)$ throughout $S$, uniformly on any closed subset of $S$. If the function $f(z)$ is analytic throughout $R_{\rho}$ but not throughout any $R_{\rho^{\prime}}, \rho^{\prime}>\rho>0$, then we have for all $t(>0)$

$$
\limsup _{M \rightarrow \infty}\left[\iint_{R_{\nu}}\left|f(z)-f_{M}(z)\right| t d S\right]^{1 / t \log M}=e^{(\nu-\rho) /(1-\rho)}, \quad 0 \leqq \nu<\rho ;
$$

and, for all $t(>0)$ if $\rho \leqq \nu<1$, and for $0<t \leqq q$ if $\nu=1$,

$$
\limsup _{M \rightarrow \infty}\left[\iint_{R_{\nu}}\left|f_{M}(z)\right|^{t} d S\right]^{1 / t \log M}=e^{(\nu-\rho) /(1-\rho)} .
$$

These equations are valid for $t=\infty$, with the understanding that for a function 
$g(z)$ analytic in $R$ the integral $\left[\iint_{R_{\nu}}|g(z)|{ }^{t} d S\right]^{1 / t}$ shall be interpreted as l.u.b. $[|g(z)|, z$ in $R$,$] .$

An analogue of Corollary 1 involving non-extremal functions is also true.

6. Problem 4. In the study of approximation in the sense of least $p$ th powers $(p>0)$ we consider a function $f_{M}(z)$ which approximates on each $C_{0}^{(J)}$ to a given function $F(z)$ of class $H_{y}$ on $C_{0}^{(j)}$ and satisfies just one prescribed condition of interpolation interior to $C_{0}^{(j)}$. A function $f(w)$ is said to be of class $H_{p}$ on $\gamma:|w|=1$ if $f(w)$ is analytic throughout the interior of $\gamma$, and if the integrals

$$
\int_{0}^{2 \pi}\left|f\left(r e^{i \theta}\right)\right| p d \theta, \quad 0<r<1
$$

are bounded. The class $H_{p}$ is additive. It is shown by F. Riesz [5, p. 91] that $f(z)$ has boundary values almost everywhere on $\gamma$ for normal approach or for approach "in angle" and that for these limit values $f\left(e^{i \theta}\right)$ the integral

$$
\int_{0}^{2 \pi}\left|f\left(e^{i \theta}\right)\right|^{p} d \theta
$$

exists. Let now $C$ be an analytic Jordan curve in the $z$-plane, whose interior is mapped onto the interior of $\gamma$ by the transformation $w=g(z), z=h(w)$. Then the function $f(z)$ is said to be of class $H_{p}(p>0)$ on $C$ if $f[h(w)]$ is of class $H_{p}$ on $\gamma$. If $f(z)$ is of class $H_{p}$ on $C$, it follows that $f(z)$ has boundary values almost everywhere on $C$ for normal approach or for approach "in angle," and that for these boundary values the integral

$$
\int_{C}|f(z)| p|d z|
$$

exists.

Functions of class $H_{p}$ have various important properties with reference to approximation:

THEOREM 6.1. If $C$ is an analytic Jordan curve and $f(z)$ is of class $H_{p}$ interior to $C$, then there exists a sequence of polynomials $p_{n}(z)$ such that we have

$$
\lim _{n \rightarrow \infty} \int_{C}\left|f(z)-p_{n}(z)\right| p|d z|=0,
$$

which implies $\lim _{n \rightarrow \infty} p_{n}(z)=f(z)$ throughout the interior of $C$, uniformly on any closed set interior to $C$. (6.1).

The boundary values of $f(z)$ almost everywhere on $C$ are to be used in

It is sufficient to prove Theorem 6.1 for the case that $C$ is the unit circle, for equation (6.1) is essentially unchanged under the map of the interior of an 
analytic Jordan curve onto the interior of the unit circle, and the possibility of approximation by polynomials in one plane implies the possibility of approximation by polynomials in the other.

The equation

$$
\lim _{r \rightarrow 1} \int_{0}^{2 \pi}\left|f\left(e^{i \theta}\right)-f\left(r e^{i \theta}\right)\right|^{p} d \theta=0
$$

is known [5, p. 93], so $f(z)$ can be approximated in the mean of order $p$ on $C$ by a function $f\left(r e^{i \theta}\right), r<1$. The function $f(r z)$ is analytic in $|z| \leqq 1$, and can be uniformly approximated in the closed interior of $C$ by a polynomial, hence can be approximated in the mean of order $p$ on $C$ by a polynomial. Standard inequalities [for example, 7, p. 93] then imply that $f(z)$ can be approximated in the mean of order $p$ on $C$ by a polynomial $p_{n}(z)$. Thus for suitable choice of the sequence $p_{n}(z)$ we have

$$
\lim _{n \rightarrow \infty} \int_{0}^{2 \pi}\left|f\left(e^{i \theta}\right)-p_{n}\left(e^{i \theta}\right)\right|^{p} d \theta=0
$$

whence by the monotonic character of the means $\int_{0}^{2 \pi}\left|f\left(r e^{i \theta}\right)-p_{n}\left(r e^{i \theta}\right)\right|^{p} d \theta$ as functions of $r$ we have [7, p. 110, Theorem 18] the conclusion of Theorem 6.1.

Theorem 6.1 applies to approximation in a single Jordan region by polynomials but obviously extends to approximation in several Jordan regions whose closures are mutually exterior. Moreover, these polynomials may be assigned auxiliary conditions of interpolation to the function approximated, by virtue of Lemma 3.1 .

G. Julia [2, p. 110] has obtained a specific formula for the function $f(z)$ of class $H_{p}$ on $C$, with $f(\alpha)=A, \alpha$ interior to $C$, and such that $\int_{C}|f(z)|^{p}|d z|$ is least. His result is used by Spitzbart $[6$, p. 344] to study degree of convergence:

THEOREM 6.2. Let $C$ be an analytic Jordan curve of the $z$-plane, and $z=\alpha$ $a$ point interior to $C$. Let $w=g(z), z=h(w)$ map the interior of $C$ conformally onto the interior of $C^{\prime}:|w|=1$ so that $z=\alpha$ corresponds to $w=0$. Among the functions of class $H_{p}, p>0$, on $C$, with $f(\alpha)=A$, the minimum of $\int_{C}|f(z)| p|d z|$ occurs for the function $F_{0}(z)=A\left\{\left[g^{\prime}(z)\right] /\left[g^{\prime}(\alpha)\right]\right\}^{1 / p}$.

Let $\left\{f_{n}(z)\right\}$ be a sequence of functions of class $H_{p}$ with $f_{n}(\alpha)=A$ and

$$
\int_{C}\left|f_{n}(z)\right| p|d z| \leqq \int_{C}\left|F_{0}(z)\right| p|d z|+\epsilon_{n}, \quad \lim _{n \rightarrow \infty} \epsilon_{n}=0 .
$$

If $D$ is any closed region interior to $C$, there is a constant $K$ depending only on $D$ such that for $z$ in $D$

$$
\left|F_{0}(z)-f_{n}(z)\right| \leqq K \epsilon_{n}^{1 / 2}
$$

If $C_{0}$ is a point set consisting of $s$ mutually exterior analytic Jordan curves, 
we say that a function $F(z)$ is of class $H_{p}$ on $C_{0}$ if it is of class $H_{p}$ on each curve.

THEOREM 6.3. Let the point set $S$ consist of the respective interiors of $s$ mutually exterior analytic Jordan curves $C_{0}^{(1)}, C_{0}^{(2)}, \cdots, C_{0}^{(s)}$. Let $F(z)$ be of class $H_{p}$ on $C_{0}$, and let the point $\alpha_{j}$ interior to $C_{0}^{(j)}$ and a functional value $A_{j}$ be given: Let $f(z)$ be the function of class $H_{p}$ on $C_{0}$ with $f\left(\alpha_{j}\right)=A_{j}$, and such that $\mu=\int_{C_{0}}|f(z)-F(z)|^{p}|d z|$ is least.

Let $f_{M}(z)$ be the function analytic and of modulus not greater than $M$ in $R$, with $f_{M}\left(\alpha_{j}\right)=A_{j}$, and for which $\int_{C_{0}}\left|f_{M}(z)-F(z)\right| p|d z|$ is least. Then the set $f_{M}(z)$ converges to $f(z)$ throughout $S$, uniformly on any closed subset of $S$. If the function $f(z)$ is analytic throughout $R_{\rho}$ but not throughout any $R_{\rho^{\prime}}, \rho^{\prime}>\rho>0$, then we have

$$
\begin{array}{ll}
\limsup _{M \rightarrow \infty}\left[\max \left|f(z)-f_{M}(z)\right|, z \text { in } \bar{R}_{\nu}\right]^{1 / \log M}=e^{(\nu-\rho) /(1-\rho)}, & 0 \leqq \nu<\rho, \\
\limsup _{M \rightarrow \infty}\left[1 . \text { u.b. }\left|f_{M}(z)\right|, z \text { in } R_{\nu}\right]^{1 / \log M}=e^{(\nu-\rho) /(1-\rho)}, & \rho \leqq \nu \leqq 1 .
\end{array}
$$

We prove the theorem for the case $s=1$.

The problem of determining the function $f(z)$ of class $H_{p}$ on $C_{0}$ satisfying the condition $f\left(\alpha_{1}\right)=A_{1}$ and such that $\int_{C_{0}}|f(z)-F(z)|^{p}|d z|$ is least is equivalent to the problem of determining the function $\Psi(z)$ of class $H_{p}$ on $C_{0}$ satisfying the condition $\Psi\left(\alpha_{1}\right)=A_{1}-F\left(\alpha_{1}\right)$ and such that $\int_{C_{0}}|\Psi(z)| p|d z|$ is least. It follows from Theorem 6.2 that the extremal function $\Psi(z)$ exists and is unique, whence the extremal function $f(z)$ exists and is unique. We have the relation $\Psi(z) \equiv f(z)-F(z)$ between these extremal functions.

By Theorem 6.1, a sequence of polynomials $p_{n}(z)$, with $p_{n}\left(\alpha_{1}\right)=f\left(\alpha_{1}\right)$, exists such that $\lim _{n \rightarrow \infty} \int_{C_{0}}\left|f(z)-p_{n}(z)\right| p|d z|=0$. For this sequence, we have, by the use of Minkowski's inequality [1, pp. 146-147],

$$
\int_{C_{0}}\left|p_{n}(z)-F(z)\right|^{p}|d z| \leqq \mu+\epsilon_{n}, \lim _{n \rightarrow \infty} \epsilon_{n}=0 ;
$$

this inequality is valid if we replace $p_{n}(z)$ by the functions $f_{M}(z)$ of Theorem 6.3. The convergence of the set $f_{M}(z)$ to $f(z)$ throughout $S$, uniformly on any closed subset of $S$, now follows from Theorem 6.2.

If $f(z)$ is analytic throughout $R_{\rho}$ but not throughout any $R_{\rho^{\prime}}, \rho^{\prime}>\rho>0$, then the functions $g_{M}(z)$ of Theorem 3.1 which coincide with $f(z)$ in the point $\alpha_{1}$ satisfy the equation

$$
\limsup _{M \rightarrow \infty}\left[\max \left|f(z)-g_{M}(z)\right|, z \text { on } C_{0}\right]^{1 / \log M}=e^{-\rho /(1-\rho)} .
$$

The extremal function $\Psi(z)$ which satisfies the condition $\Psi\left(\alpha_{1}\right)=A_{1}-F\left(\alpha_{1}\right)$ is, by Theorem 6.2, the function $\Psi(z)=\left[A_{1}-F\left(\alpha_{1}\right)\right]\left\{\left[g^{\prime}(z)\right] /\left[g^{\prime}\left(\alpha_{1}\right)\right]\right\}^{1 / p}$.

Case 1. If $A_{1}=F\left(\alpha_{1}\right)$, then $\Psi(z) \equiv 0$ on $\bar{S}$, and $f(z) \equiv F(z)$ in $\bar{S}$. Here we have 
at once, for arbitrary $\epsilon>0$, and for $K$ independent of $M$,

$$
\begin{aligned}
\int_{C_{0}}\left|f(z)-f_{M}(z)\right|^{p}|d z| & \leqq \int_{C_{0}}\left|f(z)-g_{M}(z)\right| p|d z| \\
& \leqq K e^{p(-\rho+e) \log M /(1-\rho)} .
\end{aligned}
$$

The equations (6.3) and (6.4) follow for this case by use of a lemma [generalizing 7, p. 101, Lemma; see 5] related to Cauchy's integral formula, and Lemma 2.2.

Case 2. If $A_{1} \neq F\left(\alpha_{1}\right)$, then $\Psi(z) \neq 0$ in $\bar{S}$, and we proceed to show that $g_{M}(z)$ satisfies the relation (6.2). We use the method applied by Spitzbart $\left[6\right.$, p. 342] in the special case of polynomial approximation. We write $g_{M}(z)$ $-F(z)=f(z)-F(z)+R_{M}(z)=\Psi(z)+R_{M}(z)$, where $\lim _{M \rightarrow \infty} R_{M}(z)=0$ in $\bar{S}$, and $\left[g_{M}(z)-F(z)\right]^{p / 2}=[\Psi(z)]^{p / 2}\left[1+R_{M}(z) / \Psi(z)\right]^{p / 2}$. Since $\Psi \neq 0$ in $\bar{S}$, the binomial expansion is valid if $M$ is sufficiently large, and, for given $\epsilon>0$ and $M$ sufficiently large, we have for $z$ in $\bar{S}$

$$
\begin{aligned}
\left|\left[g_{M}(z)-F(z)\right]^{p / 2}-[\Psi(z)]^{p / 2}\right| & \leqq K \max \left|R_{M}(z)\right| \\
& \leqq K e^{(-\rho+\epsilon) \log M /(1-\rho)},
\end{aligned}
$$

where $K$ is independent of $M$. Inequality (6.6) is not in its present form as strong as (6.5), and cannot be used without further manipulation to establish (6.3) and (6.4). We shall investigate more closely the integral of the first member of (6.6), interpreted in the w-plane, and thus obtain our desired results.

In the notation of Theorem 6.2 we write $\Psi(z) \equiv \Psi[h(w)] \equiv G(w)$, and $g_{M}(z)-F(z) \equiv g_{M}[h(w)]-F[h(w)] \equiv G_{M}(w)$. The function $\left\{G(w)\left[h^{\prime}(w)\right]^{1 / p}\right\}^{p / 2}$ $-\left\{G_{M}(w)\left[h^{\prime}(w)\right]^{1 / p}\right\}^{p / 2}$ is analytic on and within $\gamma$ and has the value zero in $w=0$. Hence

$$
\int_{\gamma}\left[\left\{G(w)\left[h^{\prime}(w)\right]^{1 / p}\right\}^{p / 2}-\left\{G_{M}(w)\left[h^{\prime}(w)\right]^{1 / p}\right\}^{p / 2}\right]|d w|=0 .
$$

However, by Theorem 6.2 we have $G(w) \equiv G(0)\left[h^{\prime}(0)\right]^{1 / p} /\left[h^{\prime}(w)\right]^{1 / p}$ and hence by (6.7)

$$
\begin{aligned}
\int_{\gamma}[\{G(w) & {\left.\left[h^{\prime}(w)\right]^{1 / p}\right\}^{p / 2} } \\
& \left.-\left\{G_{M}(w)\left[h^{\prime}(w)\right]^{1 / p}\right\} p / 2\right]\left\{\bar{G}(w)\left[\bar{h}^{\prime}(w)\right]^{1 / p}\right\}^{p / 2}|d w|=0 .
\end{aligned}
$$

In the $z$-plane this integral becomes

$$
\int_{C_{0}}\left\{[\Psi(z)]^{p / 2}-\left[g_{M}(z)-F(z)\right]^{p / 2}\right\}[\bar{\Psi}(z)]^{p / 2}|d z|=0
$$

whence 


$$
\begin{aligned}
\int_{C_{0}}\left|[\Psi(z)]^{p / 2}-\left[g_{M}(z)-F(z)\right]^{p / 2}\right| 2|d z| & \\
& =\int_{C_{0}}\left|g_{M}(z)-F(z)\right| p|d z|-\int_{C_{0}}|\Psi(z)|^{p}|d z| .
\end{aligned}
$$

For arbitrary $\epsilon>0$ and $K_{0}$ independent of $M$ we have by (6.6) for $M$ sufficiently large

$$
\begin{aligned}
\int_{C_{0}}\left|g_{M}(z)-F(z)\right|^{p}|d z|= & \int_{C_{0}}|\Psi(z)|^{p}|d z| \\
& +\int_{C_{0}}\left|[\Psi(z)]^{p / 2}-\left[g_{M}(z)-F(z)\right]^{p / 2}\right|^{2}|d z| \\
\leqq & +K_{0} e^{2(-\rho+\epsilon) \log M /(1-\rho)} .
\end{aligned}
$$

For the functions $f_{M}(z)$ of Theorem 6.3 instead of the $g_{M}(z)$ the latter inequality is certainly valid. Hence, by Theorem 6.2 , since $f_{M}(z)-F(z)-\Psi(z)$ $\equiv f_{M}(z)-f(z)$, we have in any closed region $\bar{S}_{1}$ in $S$

$$
\left|f(z)-f_{M}(z)\right| \leqq Q e^{(-\rho+\epsilon) \log M /(1-\rho)},
$$

where $Q$ is independent of $M$.

Lemma 2.2 now yields the conclusion of Theorem 6.3.

COROLLARY 1. Under the conditions of Theorem 6.3, let $\psi_{M}(z)$ be analytic and of modulus not greater than $M$ in $R$, and satisfy the auxiliary conditions $\psi_{M}\left(\alpha_{j}\right)=A_{j}, \alpha_{j}$ interior to $C_{0}^{(j)}$, with

$$
\lim _{M \rightarrow \infty} \int_{C_{0}}\left|\psi_{M}(z)-F(z)\right| p|d z|=\mu .
$$

Then the set of functions $\psi_{M}(z)$ converges to $f(z)$ throughout $S$, uniformly on any closed subset of $S$. If $f(z)$ is analytic throughout $R_{p}$ but not throughout any $R_{\rho^{\prime}}, \rho^{\prime}>\rho>0$, and if we also have

$$
\limsup _{M \rightarrow \infty}\left[\int_{C_{0}}\left|\psi_{M}(z)-F(z)\right|^{p}|d z|-\mu\right]^{1 / \log M} \leqq e^{-2 \rho /(1-\rho)},
$$

then the analogues of (6.3) and (6.4) with $f_{M}(z)$ replaced by $\psi_{M}(z)$ are valid.

Corollary 2. Let $q>0$ be given. Let Theorem 6.2 be modified so that $f_{M}(z)$ analytic in $R$ is required to satisfy the inequality $\int_{C_{\nu}}\left|f_{M}(z)\right| q|d z| \leqq M^{q}$ for all $\nu, 0<\nu<1$, instead of the inequality $\left|f_{M}(z)\right| \leqq M$ in $R$. Then the set $f_{M}(z)$ converges to $f(z)$ throughout $S$, uniformly on any closed subset of $S$. If the function $f(z)$ is analytic throughout $R_{\rho}$ but not throughout any $R_{\rho^{\prime}}, \rho^{\prime}>\rho>0$, then we have for all $t(>0)$ 


$$
\limsup _{M \rightarrow \infty}\left[\int_{C_{\nu}}\left|f(z)-f_{M}(z)\right| t|d z|\right]^{1 / t \log M}=e^{(\nu-\rho) /(1-\rho)}, \quad 0 \leqq \nu<\rho ;
$$

and, for all $t(>0)$ if $\rho \leqq \nu<1$, and for $0<t \leqq q$ if $\nu=1$,

$$
\limsup _{M \rightarrow \infty}\left[\int_{C_{\nu}}\left|f_{M}(z)\right| t|d z|\right]^{1 / t \log M}=e^{(\nu-\rho) /(1-\rho)} .
$$

These equations are valid for $t=\infty$, with the understanding that for a function $g(z)$ analytic in $R$ the integral $\left[\int_{C_{\nu}}|g(z)| t|d z|\right]^{1 / t}$ shall be interpreted for $t=\infty$ as l.u.b. $\left[|g(z)|\right.$, $z$ on $\left.C_{\nu}\right]$.

An analogue of Corollary 1 involving non-extremal functions is also true $\left.{ }^{4}\right)$

7. Generalizations of Problems 1-4. Problems 1-4 can be generalized by enlarging both the concepts of norm of $f_{M}(z)$ in $R$ and measure of approximation of $f_{M}(z)$ to $F(z)$ on $C_{0}$ or on $S$. For instance we may separate $C_{1}$ (assumed rectifiable) into two disjoint parts, each consisting of one or more Jordan curves, and use as the $q$ th power of the norm of $f_{M}(z)$ in $R$ the limit superior of $\left|f_{M}(z)\right|^{q}$ as $z$ in $R$ approaches one part of $C_{1}$ plus the integral of $\left|f_{M}(z)\right|^{q}$ over the remaining part of $C_{1}$. We may also consider the disjoint closed regions composing $S$, and in each of these separate regions make the appropriate assumptions (involving points of interpolation, a function $F(z)$, and a measure of approximation of $f_{M}(z)$ to $\left.F(z)\right)$ used in Problems 1-4; the present measure of approximation of $f_{M}(z)$ to $F(z)$ on $C_{0}$ or on $S$ is defined as the sum of the individual measures of approximation. Complete analogues of Theorems 3.2, 4.1, 5.1, 6.3 are then valid and can easily be formulated and established by the reader.

The use of suitable norm functions in connection both with the norm of $f_{M}(z)$ in $R$ and the measure of approximation of $f_{M}(z)$ and $f(z)$ to $F(z)$ on $C_{0}$ or on $S$ throughout the present paper presents no great difficulty and is left to the reader.

8. The complementary extremal problems. In Problems 1-4 we have assumed the norm $M$ of $f_{M}(z)$ in $R$ given and have studied the function $f_{M}(z)$ of best approximation to $F(z)$ on $C_{0}$ or on $S$, and the convergence properties of $f_{M}(z)$. The complementary problem defines a function $f_{m}(z)$ of least norm in $R$ corresponding to a given measure $m$ of approximation of $f_{m}(z)$ to $F(z)$ on

(4) A consequence of Corollary 1 is the following extension of Spitzbart's result on approximation by polynomials:

Under the conditions of Theorem 6.3 with $s=1$, the sequence of polynomials $p_{n}(z)$ of respective degrees $n$ satisfying the given auxiliary condition of best approximation to $F(z)$ on $C$ in the sense of least pth powers converges to $f(z)$ throughout the interior of $C$, uniformly on any closed set interior to $C$; if $f(z)$ is analytic in the closed interior of $C$, the sequence $p_{n}(z)$ converges maximally to $f(z)$ on $C$.

Of course this result extends at once to apply also to non-extremal polynomials. 
$C_{0}$ or on $S$; we then study the norm of $f_{m}(z)$ and the convergence properties of the set $f_{m}(z)$. To be more explicit, we define new Problems 1a-4a. Let there be given a finite number of fixed conditions of interpolation at points of $S$. Let $m(>0)$ be given, let $f_{m}(z)$ be analytic in $R$, satisfy the conditions of interpolation, approximate to $F(z)$ on $C_{0}$ or on $S$ with a measure of approximation not greater than $m$, and be such that $M_{m}=1$.u.b. $\left[\left|f_{m}(z)\right|, z\right.$ in $\left.R\right]$ is least. We study the convergence of the set $f_{m}(z)$ as $m$ approaches its minimum value. In Problem 1a the measure of approximation of $f_{m}(z)$ to $F(z)$ is $\sum_{j=1}^{s}\left[\max \left|f_{m}(z)-F(z)\right|, z\right.$ on $\left.C_{0}^{(j)}\right]$, where $F(z)$ is defined as in Problem 1; in Problem 2a the measure of approximation of $f_{m}(z)$ to $F(z)$ is $\int_{c_{0}} \mid f_{m}(z)$ $-\left.F(z)\right|^{2}|d z|$, where $F(z)$ is defined as in Problem 2; in Problem 3a the measure of approximation of $f_{m}(z)$ to $F(z)$ is $\iint_{S}\left|f_{m}(z)-F(z)\right|^{2} d S$, where $F(z)$ is defined as in Problem 3; in Problem 4a the measure of approximation of $f_{m}(z)$ to $F(z)$ is $\int_{c_{0}}\left|f_{m}(z)-F(z)\right|^{p}|d z|, p>0$, and $F(z)$ and the auxiliary conditions are restricted as in Problem 4.

In all cases the existence of the function $f_{m}(z)$ is readily proved [cf. 8, p. 483 ], provided $m$ is sufficiently large.

The function $f_{M}(z)$ of Problems 1-3 has the property that the norm of $f_{M}(z)$ in $R$ is actually equal to $M$, and the function $f_{m}(z)$ of Problems $1 \mathrm{a}-3 \mathrm{a}$ has the property that the measure of approximation is actually equal to $m$. The function $f_{M}(z)$ of Problem 4 has the property that the norm of $f_{M}(z)$ in $R$ is equal to $M$ if $p \geqq 1$, while the function $f_{m}(z)$ of Problem 4 a has the property that the measure of approximation is $m$, if $p>0$. Proofs of these statements are similar to proofs given by Walsh and Nilson [12, p. 243].

The two functions $f_{M}(z)$ and $f_{m}(z)$ in the corresponding problems are obviously connected. In Problems 1-3, and in Problem 4 when $p \geqq 1$, if $m=m_{M}$ is the least measure of approximation by a function $f_{M}(z)$ of norm not greater than $M$, then the extremal function $f_{M}(z)$ is also a function $f_{m}(z)$ of minimum norm which approximates to $F(z)$ with an error not greater than $m$; while if $M=M_{m}$ is the least norm corresponding to a function $f_{m}(z)$ of best approximation, then the extremal function $f_{m}(z)$ of Problems $1 \mathrm{a}-4 \mathrm{a}$ is also a function $f_{M}(z)$ of norm not greater than $M$ of best approximation. An immediate consequence of this property in the situation of Problems 1 and $1 \mathrm{a}$ is

THEOREM 8.1. Let the point set $S$ consist of the respective interiors of $s \mathrm{mu}$ tually exterior Jordan curves $C_{0}^{(1)}, C_{0}^{(2)}, \cdots, C_{0}^{(s)}$. Let $F(z)$ be meromorphic in $S$, continuous on $C_{0}$, and let the points $z_{k}, k=1,2, \cdots, \lambda$, (not necessarily distinct) in $S$ and the functional values $A_{k}, k=1,2, \cdots, \lambda$, be given. Let $f(z)$ be the unique function analytic in $S$, continuous in $\vec{S}$, which takes on the values $A_{k}$ in the points $z_{k}$, and such that $\max \left[|f(z)-F(z)|, z\right.$ on $\left.C_{0}^{(j)}\right]=\mu_{j}$ is least. Set $\mu=\sum_{j=1}^{s} \mu_{j}$.

Let $m(>\mu)$ be given. Let $f_{m}(z)$ be the function analytic in $R$ satisfying the auxiliary conditions, such that $\sum_{j=1}^{s}\left\{\max \left[\left|f_{m}(z)-F(z)\right|\right.\right.$, z on $\left.\left.C_{0}^{(j)}\right]\right\} \leqq m$, and for which l.u.b. $\left[\left|f_{m}(z)\right|, z\right.$ in $\left.R\right]$ is least. Then as $m \rightarrow \mu$ the set $f_{m}(z)$ con- 
verges to $f(z)$ throughout $S$, uniformly on any closed subset of $S$. If the function $f(z)$ is analytic throughout $R_{\rho}$ but not throughout any $R_{\rho^{\prime}}, \rho^{\prime}>\rho>0$, then we have

$$
\begin{aligned}
& \underset{m \rightarrow \mu}{\lim \sup }\left[\max \left|f(z)-f_{m}(z)\right|, z \text { in } \bar{R}_{\nu}\right]^{-1 / \log (m-\mu)}=e^{(\nu-\rho) / \rho}, \quad 0 \leqq \nu<\rho, \\
& \text { lim sup [l.u.b. } \left.\left|f_{m}(z)\right|, z \text { in } R_{\nu}\right]^{-1 / \log (m-\mu)}=e^{(\nu-\rho) / \rho}, \quad \rho \leqq \nu \leqq 1 . \\
& m \rightarrow \mu
\end{aligned}
$$

An analogous conclusion may still follow when a new norm for $f_{m}(z)$ in $R$ is introduced:

Corollary 1. Let $q(>0)$ be given. Let the hypothesis of Theorem 8.1 be modified so that the condition that $M_{m}=1$.u.b. $\left[\left|f_{m}(z)\right|, z\right.$ in $\left.R\right]$ is least be replaced by the condition that $M_{m}^{q}=\lim _{\nu \rightarrow 1} \int_{C_{\nu}}\left|f_{m}(z)\right| q|d z|$ is least. Then we have $\lim _{m \rightarrow \mu} f_{m}(z)=f(z)$ in $S$, uniformly on any closed set in $S$. If $f(z)$ is analytic throughout $R_{\rho}$ but not throughout any $R_{\rho^{\prime}}, \rho^{\prime}>\rho>0$, we have

$$
\begin{array}{ll}
\limsup _{m \rightarrow \mu}\left[\max \left|f(z)-f_{m}(z)\right|, z \text { in } \bar{R}_{\nu}\right]^{-1 / \log (m-\mu)}=e^{(\nu-\rho) / \rho}, & 0 \leqq \nu<\rho, \\
\lim \sup \left[\text { l.u.b. }\left|f_{m}(z)\right|, z \text { in } R_{\nu}\right]^{-1 / \log (m-\mu)}=e^{(\nu-\rho) / \rho}, & \rho \leqq \nu \leqq 1 .
\end{array}
$$

Similar conclusions for the extremal function $f_{m}(z)$ of Problems 2a, 3a, and 4a are immediately stated and proved.

9. Limit function analytic throughout $R$. Suppose Theorems 3.2, 4.1, 5.1, and 6.3 are modified so that the limit function $f(z)$ of the functions of best approximation is assumed analytic and bounded in $R$. Then whenever $M$ is sufficiently large we may choose $f_{M}(z) \equiv f(z)$, so the asymptotic properties of $f_{M}(z)$ and of the measure of approximation are obvious. An intermediate case is not trivial:

THEOREM 9.1. If the hypotheses of Theorems 3.2, 4.1, 5.1, and 6.3 are modified so that $f(z)$ is analytic throughout $R$ but not bounded in $R$, then the set $f_{M}(z)$ converges to $f(z)$ throughout $R$, uniformly on any closed subset of $R$. Moreover, equations (3.1), (4.1), (5.1), and (6.3) are replaced by

$$
\lim _{M \rightarrow \infty}\left[\max \left|f(z)-f_{M}(z)\right|, z \text { in } \bar{R}_{\nu}\right]^{1 / \log M}=0, \quad 0 \leqq \nu<1,
$$

and (3.2), (4.2), (5.2), (6.4) are replaced by

$$
\limsup _{M \rightarrow \infty}\left[\text { l.u.b. }\left|f_{M}(z)\right|, z \text { in } R_{\nu}\right]^{1 / \log M}=1, \quad 0 \leqq \nu<1,
$$

and

$$
\limsup _{M \rightarrow \infty}\left[1 . u . b .\left|f_{M}(z)\right|, z \text { in } R\right]^{1 / \log M}=e .
$$

An analogous theorem holds for the functions $f_{m}(z)$ of Theorem 8.1: 
TheOREM 9.2. If the hypothesis of Theorem 8.1 is modified so that $f(z)$ is analytic throughout $R$, then the set $f_{m}(z)$ converges to $f(z)$ throughout $R$, uniformly on any closed subset of $R$. Moreover equations (8.1) and (8.2) are replaced by

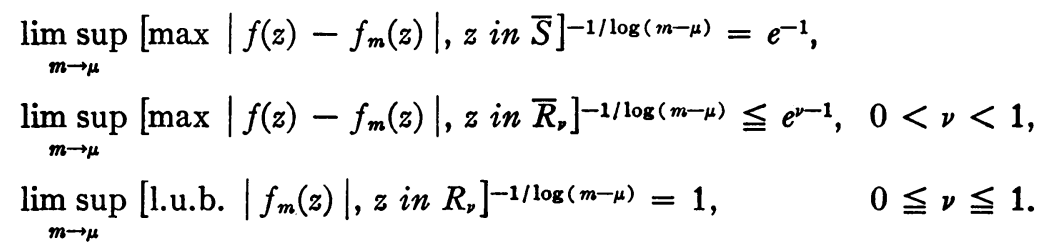

\section{REFERENCES}

1. G. H. Hardy, J. E. Littlewood, and G. P6lya, Inequalities, Cambridge, 1934.

2. G. Julia, Leçons sur la représentation conforme des aires simplement connexes, Paris, 1931.

3. H. Lebesgue, Sur le problème de Dirichlet, Rend. Circ. Mat. Palermo vol. 24 (1907) pp. 371-402.

4. E. N. Nilson and J. L. Walsh, Interpolation and approximation by functions analytic and bounded in a given region, Trans. Amer. Math. Soc. vol. 55 (1944) pp. 53-67. $87-95$.

5. F. Riesz, Über die Randwerte einer analytischen Funktion, Math. Zeit. vol. 18 (1923) pp.

6. A. Spitzbart, Approximation in the sense of least pth powers with a single auxiliary condition of interpolation, Bull. Amer. Math. Soc. vol. 52 (1946) pp. 338-346.

7. J. L. Walsh, Interpolation and approximation by rational functions in the complex domain, Amer. Math. Soc. Colloquium Publications, vol. 20, 1935.

8. - On interpolation and approximation by functions analytic and bounded in a given region, Proc. Nat. Acad. Sci. U.S.A. vol. 24 (1938) pp. 477-486.

9. - On interpolation by functions analytic and bounded in a given region, Trans. Amer. Math. Soc. vol. 46 (1939) pp. 46-65.

10. - Note on the degree of convergence of sequences of analytic functions, Trans. Amer. Math. Soc. vol. 47 (1940) pp. 293-304.

11. - Taylor's series and approximation to analytic functions, Bull. Amer. Math. Soc. vol. 52 (1946) pp. 572-579.

12. J. L. Walsh and E. N. Nilson, On functions analytic in a region: approximation in the sense of least pth powers, Trans. Amer. Math. Soc. vol. 65 (1949) pp. 239-258.

HARVARD UNIVERSiTy,

Cambridge, Mass.

Wellesley College,

Wellesley, Mass. 